\title{
Introduction-Scanning the Issue
}

I T HAS BEEN 21 years since the first fullscale conference devoted to picture archiving and communications systems (PACS) was held with the support of the International Society for Optical Engineering (SPIE). That conference was titled 1st International Conference and Workshop on Picture Archiving and Communication Systems (PACS) for Medical Applications. Led by André J. Duerinkx, who served as chair/editor, the meeting was held at Newport Beach, CA, January 18-21, 1982. The proceedings were published in two parts (Proc SPIE 1982;318[parts I and II]) in the classic yellow SPIE binding. Judith M. S. Prewitt, Sc.D., is credited with proposing the acronym PACS, which, despite proposed alternatives, remains the preference of academicians and users.

The editor of the Journal of Digital Imaging recently proposed this issue of classic articles as a way of revisiting the first and most important PACS papers. Many suggestions were proposed as to which papers should be included. As guest editor, I was given the task of selecting the papers for review by the editor. My selection method was to listen to all suggestions, prepare a list of candidates, and consult with the editor. The selection process was similar to that used when deciding who should receive a yearly bonus. The resulting selection, although certainly not without its flaws, is consistent with the goal of identifying significant published papers of early PACS workers.

The first clinical PACS paper published in the radiology literature was "An All-digital Nuclear Medicine Department,” by J. A. Parker et al.
(Radiology 1983;147:237-240). The authors described a PACS that they implemented for digital nuclear medicine images and integrated with a radiology reporting system and gamma cameras.

The earliest paper proposing the concept of PACS was "Applications of Picture Processing, Image Analysis and Computer Graphics Techniques to Cranial CT Scans," by H. U. Lemke et al. (Proceedings of the Sixth Conference on Computer Applications in Radiology and Computer/Aided Analysis of Radiological Images. Los Alamitos, CA: IEEE Computer Society Press; 1979:341-354).

During the first PACS conference, two panel discussions took place. The topic of the first was "Equipment Manufacturers' Views on PACS." This discussion was held on January 19, 1982, and was recorded and transcribed. The topic of the second panel discussion, held on January 21, was "The Medical Community's View of PACS." Both panel discussions provide interesting insights into the hopes and fears of those proposing the new concept of PACS.

The initial work of Professor H. K. Huang and his co-workers was presented in a paper titled "Digital Radiology at the University of California, Los Angeles: A Feasibility Study" (Proc SPIE 418:259-265, 1983). This paper outlined initial planning for the PACS efforts in the radiology department at UCLA. This fea-

Copyright (C) 2003 SCAR (Society for Computer Applications in Radiology)

Online publication 8 April 2003

doi: $10.1007 / s 10278-002-6031-8$ 
sibility plan was subsequently implemented to provide a filmless radiology department.

Two classic papers included in this special issue were authored by Professor G. J. Blaine, Dr. R. Gilbert Jost, and their colleagues at the Washington University School of Medicine, St. Louis, MO. "PACS Workbench at Mallinckrodt Institute of Radiology (MIR)" (Proc SPIE 1983;418[PACS II]:80-86) identified a set of basic utilities that enabled PACS design studies and experiments. This paper was presented at the second SPIE PACS conference, held in Kansas City, MO. In "Experience as a Motivation for a Campus-wide Picture Network" (SPIE 1986;626[PACS]:549-556), the same group discussed the development of an electronic picture network within an application-area context. Sites to be served by the broadband network included radiology, cardiology PET, and neurology PET, among other imaging modalities.

The paper from Seshadri and other researchers at the University of Pennsylvania, "Prototype Medical Image Management System (MIMS) at the University of Pennsylvania: Software Design Considerations-1987' (Proc SPIE 1987;767:793-800) described prototype PACS hardware and software that began in 1981.

Kim and the group of PACS researchers from the Department of Radiology and Electrical Engineering at the University of Washington presented "Development of a PC-based Radiological Imaging Workstation" (Proc SPIE 1988;914:1257-1264). The developed softcopy display workstation used a standard IBM PC/AT computer with a custom-designed image processor implemented on two IBM PC/AT boards.

In a unique paper, three university groups described the Digital Imaging Network, a federally funded evaluation project. Mun et al. presented "Experience with Image Manage- ment Networks at Three Universities: Is the Cup Half-empty or Half-full?"' (Proc SPIE 1989;1063:194-201).

A group of researchers at Georgetown University Hospital have worked on the development of all aspects of PACS. The development of their workstations was described by Horii et al. in the paper titled "Environmental Designs for Reading from Imaging Workstations: Ergonomic and Architectural Features" (Proc SPIE 1989;1091:172-178). This group also developed the integration of a hospital information system and radiology information system interface with a PACS, "Assessment of the Integration of a HIS/RIS with a PACS" (Proc SPIE 1990;1234:391-397).

An often cited reference paper on workstations from Arenson and colleagues at the University of Pennsylvania is included here: "The Digital Imaging Workstation" (Radiology 1990;176:303-315).

Our last and most recent classic paper is from the research group at the Veterans Affairs Maryland Health Care System and the University of Maryland School of Medicine (Baltimore, MD), dealing with the issue of workflow applied to PACS. Siegel and Reiner contributed "Work Flow Redesign: The Key to Success When Using PACS" (AJR American Journal of Roentgenology 2002;178:563-566).

I am grateful to Leigh Burke and Anna Marie Mason for providing staff support and to all the authors for their assistance in developing a classic issue of $J D I$. I thank Steve Horii for his efforts on behalf of $J D I$ and all his efforts in making digital imaging and communications in medicine (DICOM) standards effective tools for PACS.
Samuel J. Dwyer III, Ph.D., Guest Editor 